\title{
Manejo Odontológico del Síndrome de Moebius
}

\section{Resumen}

El síndrome de Möebius es una alteración congénita caracterizada por parálisis de los pares craneales debido a una atrofia de sus núcleos, principalmente se ven afectados el nervio facial y el nervio abducens causando parálisis facial y limitación del movimiento ocular. Otros nervios craneales también se ven afectados lo que trae anomalías dentales y esqueletales. Puede a su vez, estar asociado a malformaciones de las extremidades superiores e
Rosa M. Stabile-Del Vecchio ${ }^{1}$, Mariana C. Morales-Chávez $z^{2}$

Relato de caso

\section{Tratamento dentário da Sindrome de Möebius}

\section{Resumo}

A síndrome de Möebius é uma alteração congénita caracterizada por a paralisia dos pares cranianos, devido à atrofia dos seus núcleos. O nervo facial e o nervo abducente são os mais afetados, causando paralisia facial e limitação de movimento do olho. Outros nervos cranianos também são afetados, causando anomalias dentárias e esqueléticas. Ao mesmo tempo, a síndrome pode ser associada a malformações das extremidades superiores e inferiores, como inferiores como sindactilia y pie equinovaro. Su etiología es aún desconocida, pero se relaciona a un problema vascular que afecta el desarrollo de los núcleos de los pares craneales. El objetivo de esta investigación es describir las características del Síndrome de Möebius y reportar 3 casos clínicos que acudieron a consulta odontológica.

Palabras claves: Síndrome de Moebius, salud bucal, maloclusión, discapacidad intelectual, nervio facial, parálisis facial. 


\section{Case Report}

\section{Dental treatment for Möebius Syndrome}

\begin{abstract}
The Moebius syndrome is a congenital disorder characterized by a paralysis of the cranial pairs, due to an atrophy of their nuclei. The facial nerve and the abducens nerve are the most affected, causing facial paralysis and limitations of the ocular movement. Other cranial nerves are also affected, which consequence is dental and skeletal anomalies. At the same time, it can be associated to malformations of upper and lower extremities, such as webbed
\end{abstract}

\section{Introducción:}

El síndrome de Moebius es una alteración congénita descrita por primera vez en 1880 por von Graefe y Saemisch y de una forma más completa en 1888; posteriormente fue descrita por Moebius en 1892. La tasa de prevalencia es de 1/150.000 nacidos vivos, sin que esto disminuya la importancia de su reconocimiento. En cuanto a la prevalencia de género afecta por igual a ambos sexos. ${ }^{1-11}$

En este síndrome se ven afectados dos importantes nervios craneales, el abducens (VI) y el nervio facial (VII), los cuales no están bien desarrollados, causando parálisis facial y limitación del movimiento ocular incluso estrabismo convergente. ${ }^{1-2,4-15}$

Su etiología es desconocida, pero se conoce que se debe a una atrofia del núcleo craneal lo que causa un problema vascular en el desarrollo inicial del embrión, donde existe una ausencia de desarrollo de los núcleos de los pares craneales VI y VII por una disrupción o interrupción del suministro san-

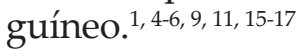

De igual manera se ha relacionado esa destrucción del núcleo de los nervios craneales a causas externas como infecciones, consumo de drogas o de medicamentos; recientemente se ha estable- fingers and toes and club foot. Its etiology is still unknown, but is related to a vascular problem that affects the development of the nuclei of the cranial pairs. The objective of this research is to describe the characteristics of the Moebius Syndrome, and report 3 clinical case studies that attended a dental consultation.

Key words: Mobius syndrome, oral health, malocclusion, intellectual disability, facial nerve, facial paralysis.

cido que la exposición al misoprostol durante la gestación tiene relación con la aparición del síndrome de Moebius. ${ }^{1,6,8,11,15}$

El compromiso de los pares craneales VI y VII produce características particulares del síndrome como cierre incompleto de los párpados durante el sueño, úlceras corneales, escurrimiento permanente de saliva, estrabismo convergente y ausencia de movimientos de los músculos de la expresión facial (aún durante el llanto). De igual forma, pueden estar comprometidos los nervios IX (glosofaríngeo), X (nervio vago o neumogástrico), XII (nervio hipogloso) y III (nervio motor ocular común), entre los menos afectados se encuentra el IV (nervio patético), VIII (nervio auditivo), V (nervio trigémino), XI (nervio accesorio o espinal) y I (nervio olfatorio). ${ }^{1,7-9,10,13,15}$

Entre sus manifestaciones clínicas y orales se encuentran: la baja implantación del pabellón auricular, anomalías de los lóbulos de los oídos, fascies inexpresivas (falta de sonrisa-inexpresión facial), micrognatia, microstomía, sobrecrecimiento del maxilar lo que conlleva a una mordida profunda, un paladar profundo y atrofia de la lengua. Se produce igualmente dificultad para deglutir y para hablar por problemas de pronunciación, reflujo gastroesofágico, hipoglosia, dificultades para succionar, crisis de broncoespasmo, disartria, disfagia 
y vaciamiento gástrico lento. Por otro lado, se observan de problemas dentales tempranos como caries rampante y gingivitis por autoclisis inapropiada y dieta inadecuada. ${ }^{1-8,10-16,18}$

La limitada apertura bucal, ocasiona problemas de alimentación, y en consecuencia una nutrición inapropiada; se puede presentar una incompleta formación del esmalte (hipoplasia) que hace que los dientes sean más vulnerables a las caries. Así mismo, se puede presentar mordida abierta anterior, debido a que los dientes anteriores no ocluyen con su antagonista y por la dificultad de cerrar o tragar, lo que además trae un deficiente crecimiento de la mandíbula y colapso transversal de los maxilares; la falta de buen sellado labial provoca sequedad, irritación e inflamación con facilidad causando enfermedad periodontal. ${ }^{1,5,8,12-13,18}$

Se ha reportado asociación con anormalidades de las extremidades y los dedos de los pies como sindactilia y braquidactilia; así como con la aparición de labio y paladar hendido; anormalidades en la pared del tórax o ausencia de los músculos pectorales (síndrome de Poland), y a la presencia de anquiloglosia. ${ }^{1-2,6-8,12,15}$

El diagnóstico se basa en cinco características del síndrome, en las que se encuentran facies inexpresivas, babeo, cierre incompleto del párpado, estrabismo convergente y dificultad para deglutir y hablar. ${ }^{1}$

Es importante el diagnóstico temprano para disminuir las secuelas mediante rehabilitación y terapias enfocadas en el área de la fonación y deglución. El tratamiento es fundamentalmente quirúrgico orientado a la corrección del cierre palpebral, pliegue epicántico y corrección de la ptosis labial. ${ }^{1,6,11}$ Además de ello, en el tratamiento bucodental, el uso de la ortopedia funcional y la mioterapia deben ser consideradas en el manejo del paciente.

El objetivo de esta investigación es describir las características del Síndrome de Möebius y reportar 3 casos clínicos que acudieron a consulta odontológica en un centro de Pacientes Especiales y que fueron evaluados y tratados luego de la firma del consentimiento informado por parte de sus repre- sentantes en el cual se explicó claramente los tratamientos a realizar a cada paciente, dándoles la libertad de retirarse en el momento que desearan. Así mismo, se obtuvo el aval bioético por parte del comité de bioética de la Universidad de Valencia. Reporte de Casos Clínicos:

Caso 1: Paciente femenina de 4 años de edad, portadora del Síndrome de Möebius, producto de segunda gesta de un matrimonio joven no consanguíneo. Dentro de sus antecedentes prenatales, el embarazo transcurrió con normalidad hasta la semana 20 en que se detectó una hidrocefalia leve por lo que se realizó amniocentesis que arrojó como resultado cariotipo fetal 46, XX (normal); en posteriores controles la hidrocefalia disminuyó siendo el eco cerebral fetal totalmente normal a las 38 semanas. Tras el parto presentaba buena vitalidad pero posteriormente al intentar iniciar la lactancia materna, se observó que no succionaba y la movilidad espontánea no era muy intensa. Debido a ello, el pediatra indicó la realización de una electromiografía facial determinándose falta total de conducción en el territorio del nervio facial de forma bilateral. Al examen clínico se evidenció falta de movilidad de musculatura facial lo que le otorga la típica facies de máscara por parálisis bilateral completa, así como sindactilia cutánea de falange proximal de 2do-3er dedo de pie izquierdo, existiendo además una pequeña deformidad en el tarso de ese pie, y estrabismo convergente. Al examen intrabucal se presenta mayor desarrollo transversal de maxilar, mordida profunda y micrognatia. Así mismo, se diagnosticó traumatismo dental en el 5.1 y del 6.1 con fractura del borde incisal de un año de antigüedad originado por una caída, presencia de caries en 7.1, 8.1 y 8.2, apiñamiento inferior, aumento de volumen y cambio de coloración gingival. La paciente recibió tratamiento de las caries con resinas compuestas, así como la reconstrucción de las fracturas causadas por los traumatismos. Igualmente se le realizó tratamiento periodontal mediante tartrectomía ultrasónica y enjuagues de clorhexidina al $0.12 \%$ por dos semanas para controlar la gingivitis y se planificó tratamiento con ortopedia funcional (Fig $1,2,3)$. Igualmente se realizó profilaxis y aplicación tópica de flúor, citándola para su cita control en 3 meses considerando su riesgo cariogénico. 


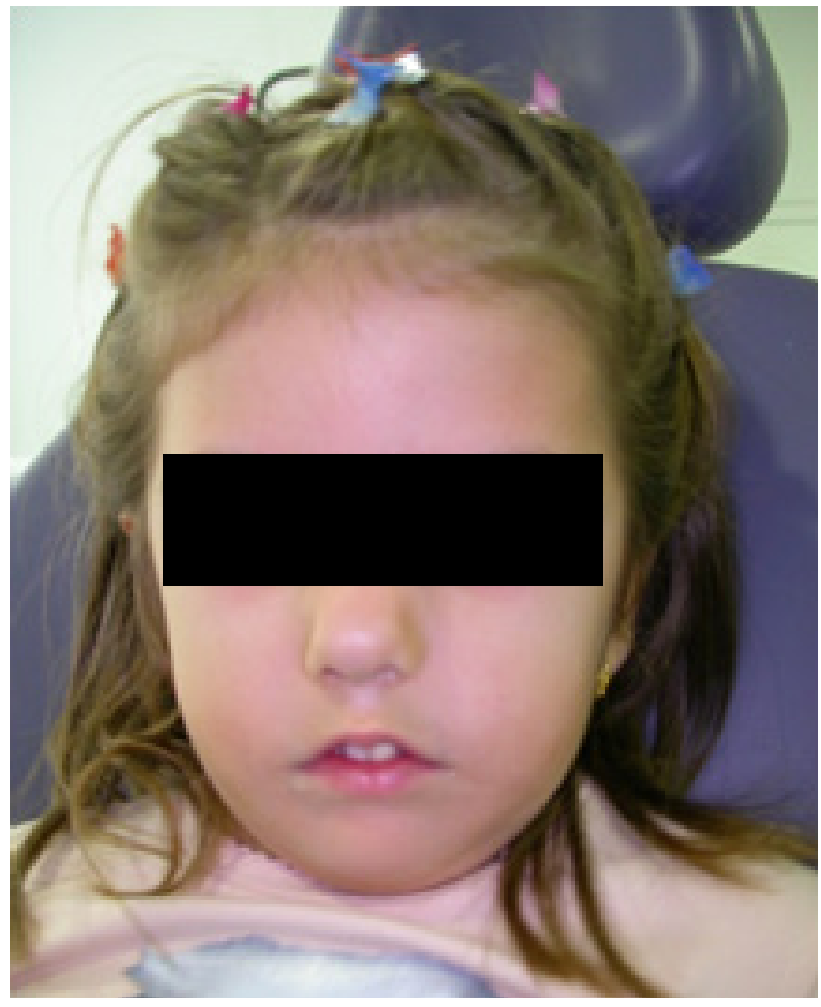

Fig. 1. Esta fotografía muestra las características de una niña de 4 años con Síndrome de Möebius.

Caso 2: Paciente femenina de 9 años de edad, segunda hija de un matrimonio no consanguíneo, portadora del Síndrome de Moebius. En cuanto

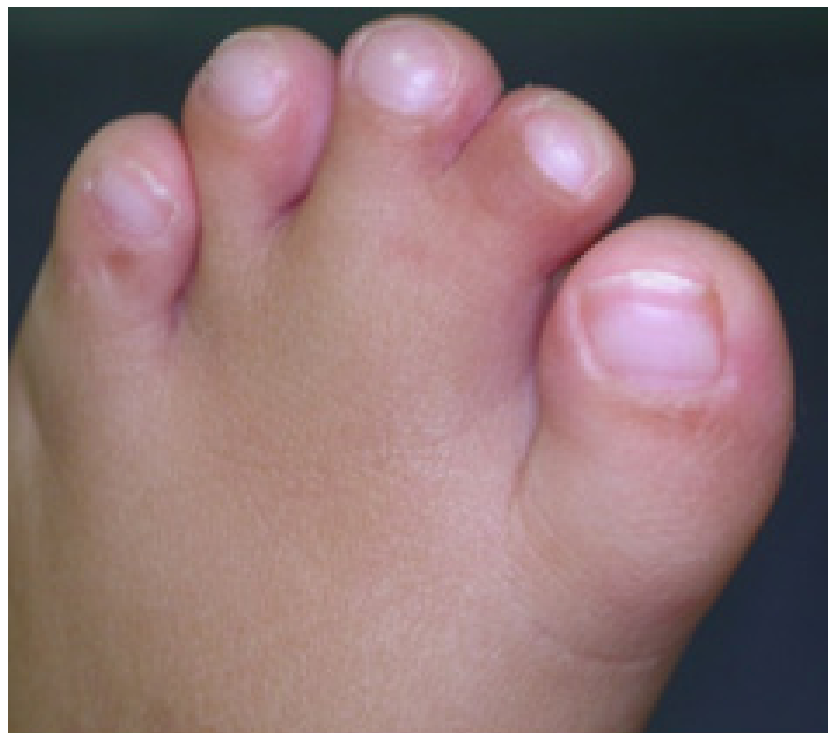

Fig. 3. Esta imagen muestra la malformanción en los dedos de los pies.

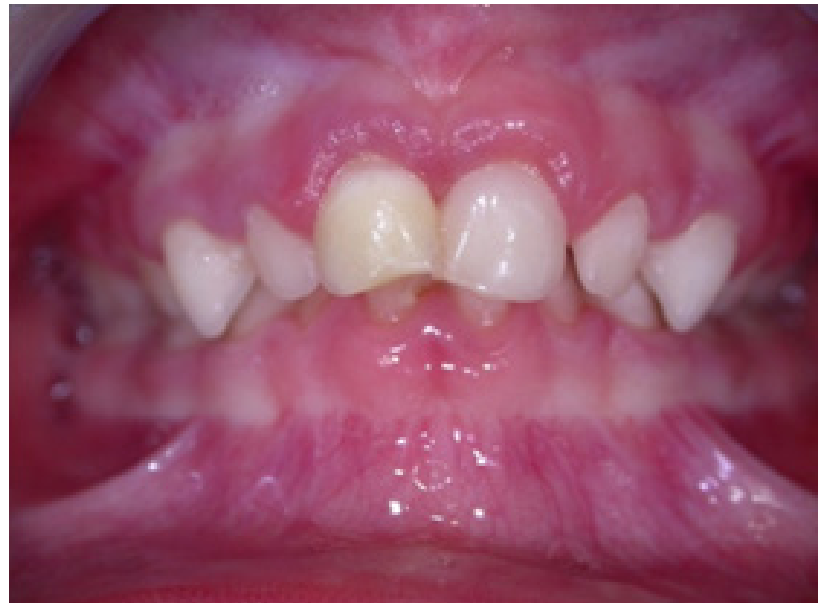

Fig. 2. Esta fotografía muestra la dentición en oclusión faciales mostrando mordida progunda y malposición.

a sus antecedentes personales es producto de una gestación múltiple, donde la madre tuvo amenaza de parto prematuro a los 2 meses perdiendo dos de los fetos. Al examen clínico se evidenció parálisis facial bilateral (predominio izquierda) con desviación de la comisura bucal a la derecha y comisura bucal izquierda caída, además de babeo secundario. No presenta alteraciones en la deglución ni en la fonación, y se evidencia ptosis palpebral (cierre palpebral incompleto), estrabismo convergente y diplopía, pie equinovaro residual que altera la marcha. Al examen intrabucal se observa atrofia y asimétrica lingual principalmente del lado derecho (atrofia hemilengua derecha), presencia de caries múltiples, sobremordida posterior izquierda por micrognatia, aumento de volumen y cambio de coloración gingival. A la paciente se le realizó tratamiento periodontal con tartrectomía ultrasónica y se colocaron resinas en posterior a la eliminación de la caries, así como tratamiento de ortopedia funcional para corregir la oclusión (Figure 4,5,6).

Caso 3: Paciente femenina de 5 años de edad con diagnóstico temprano de Síndrome de Moebius. Entre los antecedentes médicos su madre refiere que es producto es una segunda gesta entre padres sanos no consanguíneos. Al examen clínico se observa la presencia de estrabismo convergente, pie equinovaro y parálisis facial bilateral. Al examen intrabucal se evidenció traumatismo en 6.1 con fractura de esmalte originada por un golpe, mordida profunda, micrognatia, hipertro- 


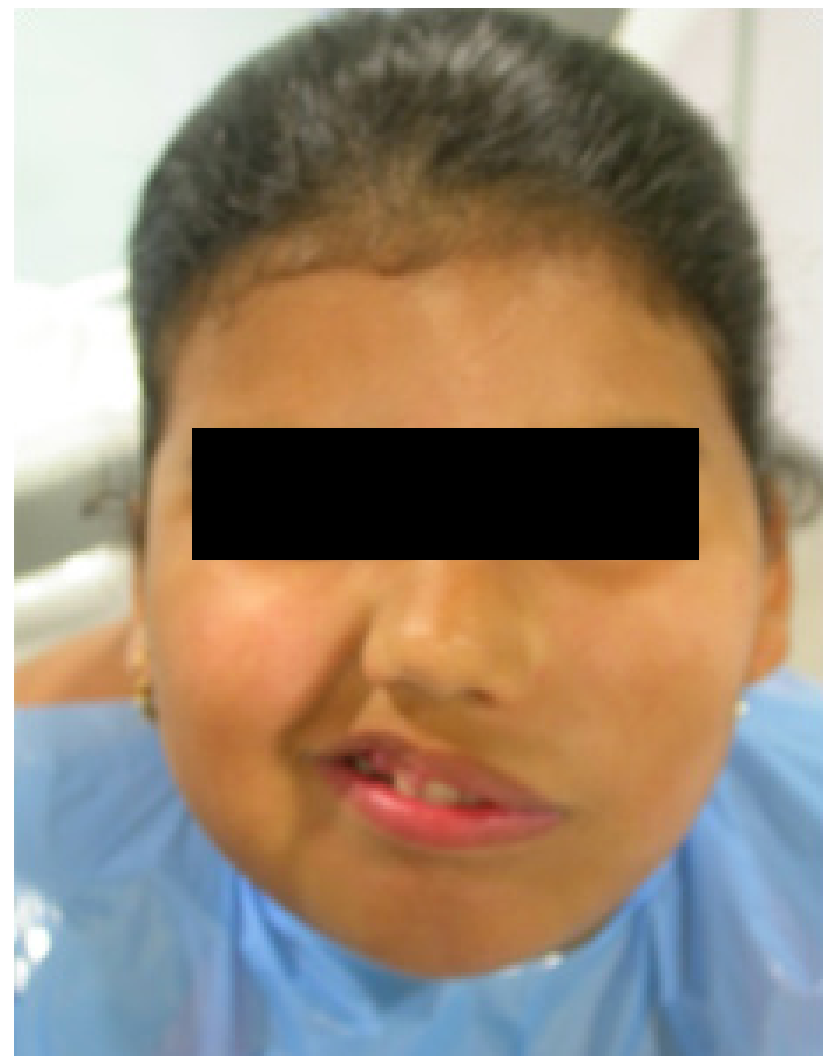

Fig. 4. Esta fotografía muestra las características faciales que incluyen páralisis facial con predominio.

fia amigdalina, caries multiples y aumento de volumen y cambio de coloración gingival generalizado. Por otro lado, se diagnosticó atrofia en línea media de lengua. Se realizó

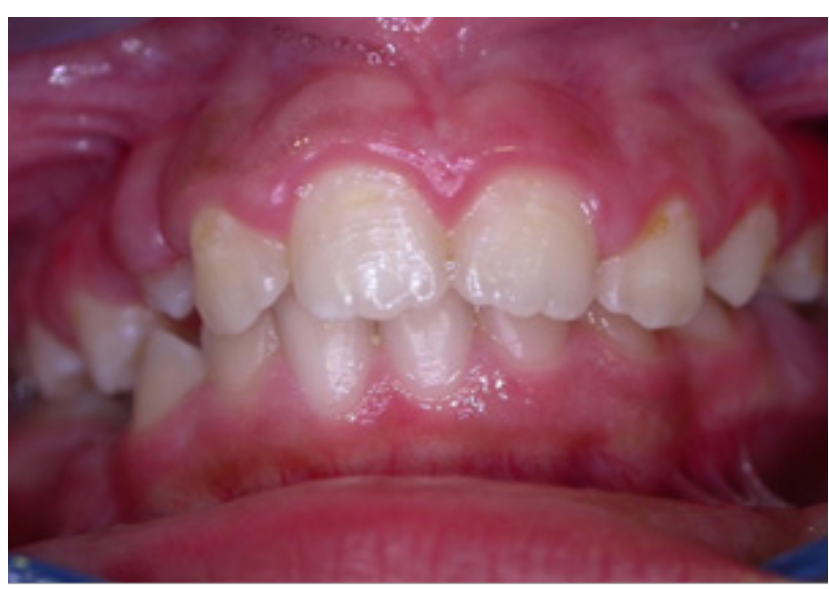

Fig. 6. Esta fotografía muestra la dentición en oclusión, la malposición. Las líneas medias del maxilar y la mandíbula no coinciden.

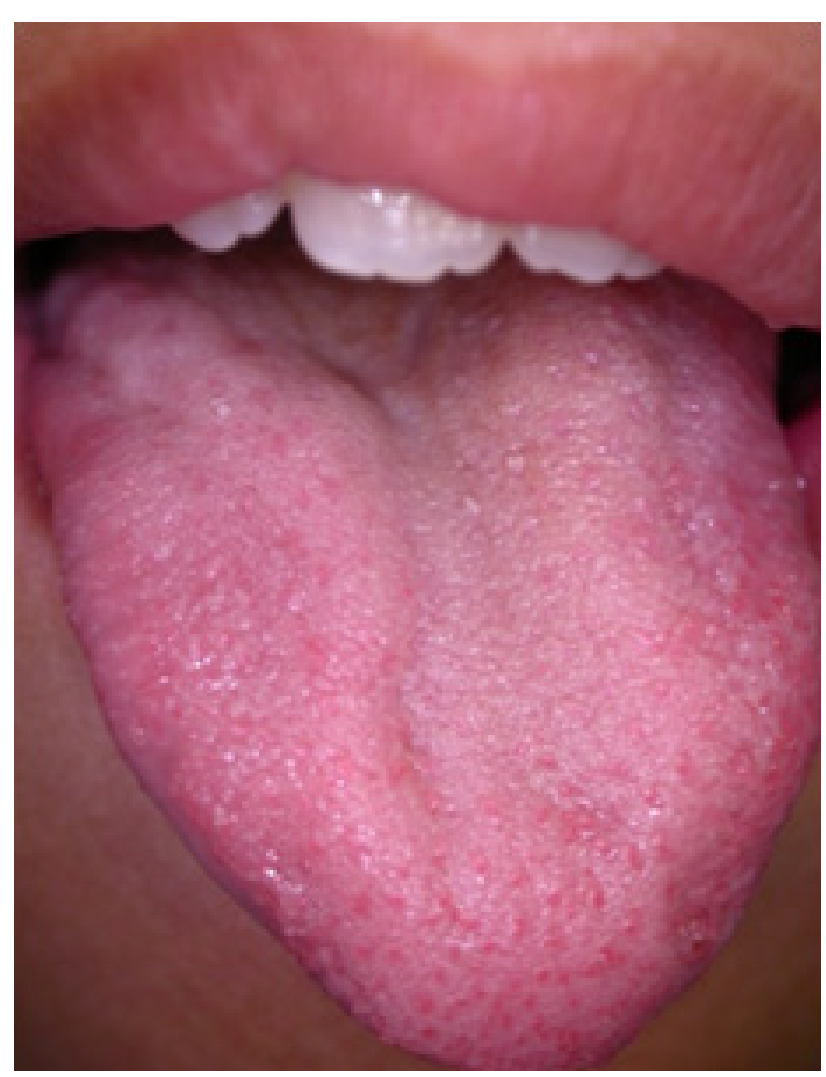

Fig. 5. Esta imangen muestra la asimetría lingual.

exodoncia del 5.5 debido a la imposibilidad de restaurar por la gran extensión de la caries. Posterior a ello, se colocó un mantenedor de espacio. Así mismo, se eliminaron las caries restantes y los dientes fueron restaurados con resinas. El tratamiento periodontal se realizó con ultrasonido para el control de la gingivitis y se colocaron pistas indirectas planas para la corrección de la mordida profunda (Fig 7,8).

\section{Discusión:}

La importancia de esta investigación se basa en la descripción de tres casos clínicos con Síndrome de Moebius, en el que cada uno presenta sus características y detalles del manejo de los pacientes y sus tratamientos específicos (restaurador, periodontal y de oclusión), con el fin de mejorar su calidad de vida. 


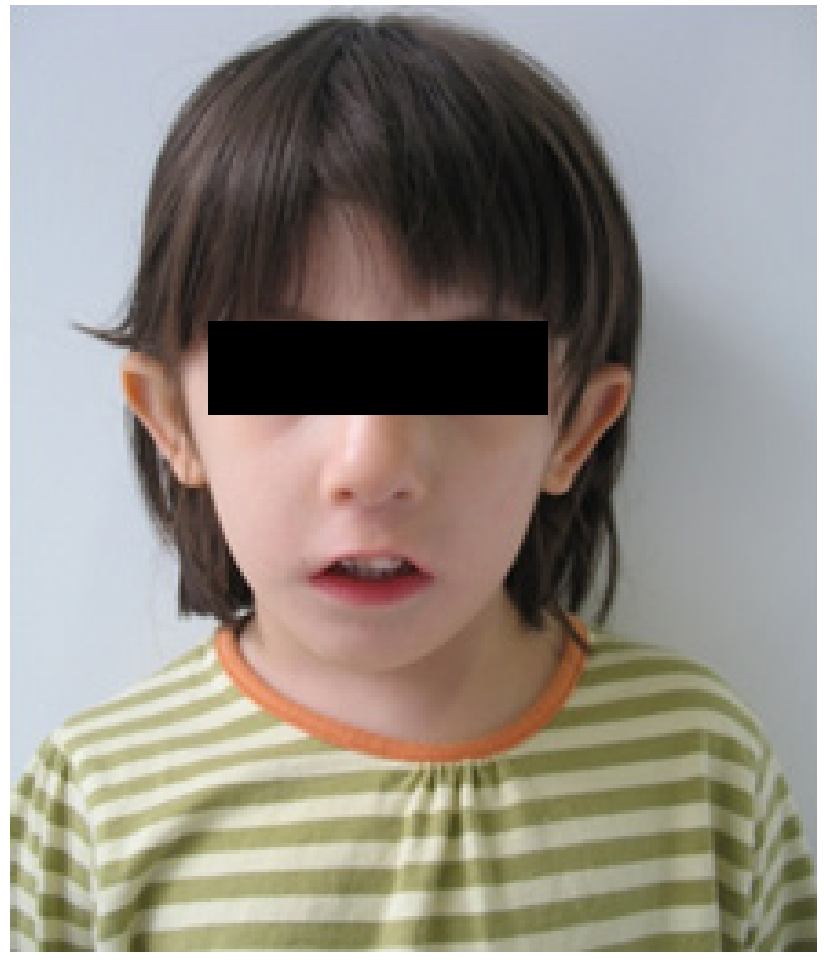

Fig. 7. Esta imagen muestra las características faciales del paciente que incluye asimetría facial.

Como ocurre en la mayoría de los pacientes con síndrome de Moebius, donde la etiología es desconocida, solo en pocos casos se debe a alteraciones genéticas, factores externos como infecciones o al uso de medicamentos como el misoprostol y/o cocaína además de traumas durante el embarazo. ${ }^{2-6,}$ 8-9, 11, 13-15 En los casos descritos, uno de ellos mostró problemas durante el embarazo como la hidrocefalia, el otro fue producto de un embarazo múltiple donde la madre perdió dos de los fetos en un aborto espontáneo, y el tercer caso no tenía ningún antecedente.

Las características clínicas y evolución de los casos coinciden en gran medida con lo descrito en la literatura. Se presenta parálisis facial bilateral de forma completa y en uno de los casos con predominio del lado izquierdo, de igual forma que estrabismo convergente y limitación del movimien-

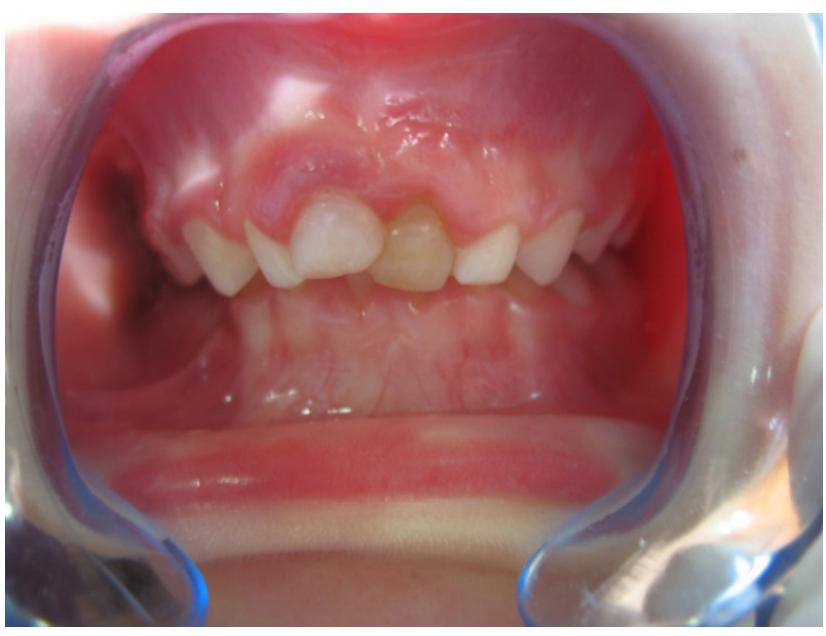

Fig. 8. Esta imagen muestra la severa mordida profunda.

to ocular lo que genera ptosis palpebral; y presencia de otras alteraciones como malformaciones de las estructuras orofaciales y anomalías de las extremidades. ${ }^{3-4,}$ 6-8, 10-11, 13, 15 Las alteraciones músculo esqueléticas que se evidenciaron en los casos reportados fueron pie equinovaro y en miembros inferiores sindactilia. ${ }^{6,8}$

En la literatura se reporta que existe presencia de retraso mental asociado al síndrome de Möebius entre $10 \%$ y $75 \%$ de los casos, $3,7-8,13$ en esta serie de 3 casos no se evidenció presencia de discapacidad intelectual asociada.

Es frecuente encontrar maloclusiones esqueléticas clase II y III, además de mordidas abiertas anteriores y mordidas profundas, donde la mayor prevalencia que se evidencia en la literatura es mordidas abiertas. ${ }^{4-5,7-8,12-14}$ En los casos reportados, la maloclusión más prevalente fue mordida profunda, debido al mayor desarrollo del maxilar y la micrognatia. Dicha micrognatia se presenta en la mayoría de los casos por un déficit neuromuscular que limita el movimiento del crecimiento inicial de la mandíbula durante los primeros movimientos como la succión. Algunos autores recomiendan el uso temprano de aparatos de ortopedia para prevenir o tratar las maloclusiones y la glosoptosis. ${ }^{5}$ 
La presencia de asimetrías y de áreas atróficas de la lengua también es característica del síndrome y en los tres casos se evidenció la presencia de esta característica. ${ }^{6-8,12-13}$

En la mayoría de estos pacientes la apertura bucal se encuentra disminuida en un rango de 15 a 30mm generalmente, lo que coincidió con lo evidenciado en estos pacientes. ${ }^{4-5,12,14}$

El tratamiento odontológico de los pacientes con síndrome de Möebius es limitado por la falta de movilidad y el deficiente desarrollo de la musculatura de la cavidad oral y del área orofacial, dificultando así la práctica de una higiene oral adecuada. Los problemas dentales en este síndrome son consecuencia de la incapacidad del niño para realizar una correcta higiene bucal, además de las parafunciones de la lengua, la hipotonia labial, la falta de autoclisis y a la ausencia de sellado perioral; se asocia también una dieta inadecuada, siendo ésta pastosa y con mayor proporción de carbohidratos fermentables. Debido a ello, suelen presentar mayor prevalencia de caries y enfermedad periodontal por lo que necesitan un control odontológico riguroso donde se realicen profilaxis y aplicaciones tópicas de flúor como medidas preventivas para la caries y la enfermedad periodontal, además de realizar una acción educativa de las personas re- sponsables del paciente que permita mejorar su salud bucal. ${ }^{5-8,12-15}$

Como resultado, es muy importante la educación del cuidado oral a los pacientes con este síndrome y sus padres, así como, la aplicación de los métodos de prevención tales como, el cepillado dental, el uso de un enjuague bucal e hilo dental, el fluoruro tópico y un control dental periódico por un especialista.

\section{Conclusión:}

Existen pocos casos reportados en la literatura sobre el tratamiento dental de los pacientes con Síndrome de Moebius por lo cual este artículo reviste gran importancia. En los casos reportados se obtuvo un resultado exitoso del tratamiento de las tres pacientes, lo cual se evidenció en las citas controles de cada una. El manejo odontológico de este tipo de pacientes se hace complejo debido a la condición física y cognitiva de los mismos. Debido a ello, una correcta evaluación y diagnóstico de las necesidades reales de cada paciente en particular permitirán una mejor planificación del caso y por ende se lograrán resultados más óptimos.

\section{Referencias bibliográficas.}

1. Morales-Chávez M, Ortiz-Rincones MA, Suárez-Gorrin F. Surgical techniques for smile restoration in patients with Möbius syndrome. J Clin Exp Dent 2013; 5(4): e203-7.

2. Ahmad M, Silvera-Redondo C, Hamdan-Rodríguez M. Moebius-Poland syndrome: a case report. Sal Uni 2012; 28(1): 171-7.

3. Rizos M, Negrón RJ, Serman N. Möbius Syndrome with dental involvement: a case report and literatura review. Clef Palate-Craniofacial Journal 1998; 35(3): 262-8.

4. Sjögreen L, Andersson-Norinder J, Jacobsson C. Development of speech, feeding, eating and facial expression in Möbius sequence. Int J Pediatr Otorhinolaryngol 2001; 60(3): 197-204.

5. Magalhães MHCG, Araújo LCA, Chiaradia CC, Fraige A, Zamurano MT, Mantesso A. Early dental management of patients with Mobius syndrome. Oral Diseases 2006; 12(6): 533-6.

6. Srinivasa-Raju M, Suma GN, Prakash R, Goel S. Moebius Syndrome: a rare case report. JIAOMR 2011; 23(3): $267-70$.

7. Shashikiran ND, Subba-Reddy VV, Patil R. Moebius Syndrome: a case report. J Indian Soc Ped Prev Dent 2004; 22(3): 96-9.

8. Scarpelli AC, Brener-Vertchenko T, Silva-Resende VL, Silva-Castilho L, Martins-Paiva S, Almeida-Pordeus I. Móbius Syndrome: a case with oral involvement. Clef Palate-Cranio Jour 2008; 45(3): 319-24.

9. Ashrafi MR, Hussain I, Zamani MA. Möebius syndrome and corpus callosum agenesis: a case report. Act Med Iranic 2006; 44(6): 432-4. 
10. Kaneria MV. Moebius Syndrome. JIACM 2006; 7(1): 53-4.

11. Carta A, Mora P, Neri A, Favilla S, Sadun AA. Ophthalmologic and systemic features in möbius syndrome: an Italian case series. Ophthalmo 2011; 118(8): 1518-23.

12. Domingos AC, Lopes SLCP, Almeida SM, Boscolo FN, Whaites EJ. Poland-Moebius syndrome: a case with oral anomalies. Oral Diseases 2004; 10: 404-7.

13. Strömland K, Sjögreen L, Miller M, Gillberg C, Wentz E, Jonhansson M, Nylén O, Danielsson A, Jabcosson C, Andersson J, Fernell E. Möbius sequence: a Swedish multidiscipline study. Euro J Paedriatr Neurol 2002; 6: 35-45.

14. De Serpa-Pinto MVX, De Magalhães MHCG, Nunes FD. Moebius syndrome with oral inveolvement. Inter J Paedriatr Dentist 2002; 12: 446-9.

15. Ouanounou S, Saigal G, Birchansky S. Möbius Syndrome. Am J Neuroradiol 2005; 26: 430-2.

16. Bonanni P, Guerrini R. Segmental facial myoclonus in Moebius Syndrome. Movement Disorders 1999; 14(6): 1021-4.

17. Van der Zwaag B, Verzijl HTFM, Beltran-Valero D, Schuster VL, van Bokhoven H, Kremer H, van Reen M, Wichers

GH, Brunner HG, Padberg GW. Mutation analysis in the candidate Möbius syndrome genes PGT and GATA2 on chromosome 3 and EGR2 on chromosome 10. J Med Genet 2002; 39: e30-2.

18. Jennings JE, Costigan C, Reardon W. Moebius sequence and Hypogonodatrophic Hypogonadism. Am J Med Genet 2003; 123A: 107-10.

Recibido: 01-05-16

Aceptado 23-07-16

Correspondencia: Mariana Morales Chávez.

Av. Trinidad con calle Caracas.

Centro Profesional Vizcaya, piso 3 ofic 37.

Caracas, Venezuela,

macamocha@hotmail.com 